\title{
Expression and prognostic significance of Src family members in renal clear cell carcinoma
}

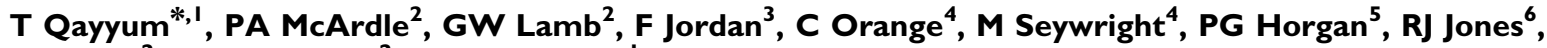 G Oades ${ }^{2}$, MA Aitchison ${ }^{2}$ and J Edwards '}

'Unit of Experimental Therapeutics, Institute of Cancer, College of MVLS, University of Glasgow, Western Infirmary, Glasgow GI I 6NT, UK; ${ }^{2}$ Department of Urology, Gartnaval General Hospital, Glasgow, UK; ${ }^{3}$ Division of Developmental Medicine Reproductive and Maternal Medicine, Faculty of Medicine, Western Infirmary, Glasgow, UK; ${ }^{4}$ Department of Pathology, Western Infirmary, Glasgow, UK; ${ }^{5}$ School of Medicine, College of MVLS, University of Glasgow, Royal Infirmary, Glasgow, UK; ${ }^{6}$ Beaston West of Scotland Cancer Clinic, Gartnaval General Hospital, Glasgow, UK

BACKGROUND: The aim of this study was to determine whether Src family kinases (SFK) are expressed in renal cell cancer and to assess their prognostic significance.

METHODS: mRNA expression levels were investigated for the 8 SFK members by quantitative real-time PCR in 19 clear cell cancer tissue samples. Immunohistochemical staining was utilised to assess expression of Src kinase, dephosphorylated Src kinase at $Y^{530}$ $\left(\operatorname{Src} \mathrm{Y}^{530}\right)$, phosphorylated Src at $Y^{419}\left(\operatorname{Src} Y^{419}\right)$ and the downstream focal adhesion kinase (FAK) marker at the $Y^{861}$ site (FAK $Y^{861}$ ) in a cohort of 57 clear cell renal cancer specimens. Expression was assessed using the weighted histoscore method.

RESULTS: Src, Lyn, Hck, Fgr and Fyn were the most highly expressed in renal cancer. All members were more highly expressed in T2 disease, and furthermore expression levels between T2 and T3 disease showed a significant decrease for Lck, Lyn, Fyn, Blk and Yes $(P=0.032)$. Assessment of membrane, cytoplasm and nuclear expression of Src kinase, SrcY ${ }^{530}$ and SrcY ${ }^{419}$ were not significantly associated with cancer-specific survival. High expression of cytoplasmic FAK $Y^{861}$ was associated with decreased cancer-specific survival $(P=0.001)$. On multivariate analysis, cytoplasmic FAK $Y^{861}$ was independently associated with cancer-specific survival (hazard ratio $3.35,95 \% \mathrm{Cl}$ 1.40-7.98, $P=0.006)$.

CONCLUSION: We have reported that all SFK members are expressed in renal cell carcinoma. The SFK members had their highest levels of expression before the disease no longer being organ confined. We hypothesise that these SFK members are upregulated before the cancer spreading out-with the organ and given that Src itself is not associated with cancer-specific survival but the presence of FAK $Y^{861}$, a downstream marker for SFK member activity is associated with decreased cancer-specific survival, we hypothesise that another SFK member is associated with decreased cancer-specific survival in renal cell cancer.

British Journal of Cancer (2012) 1 07, 856-863. doi:I0.I038/bjc.20I2.3I4 www.bjcancer.com

Published online 19 July 2012

(c) 2012 Cancer Research UK

Keywords: Src kinase; Src; FAK; renal cancer

In the United Kingdom alone, approximately 8000 new cases of renal cancer are diagnosed each year and 3800 die of their disease (www.cancerresearchuk.org, 2011). Overall survival is poor, even for those patients who undergo resection; the estimated 5-year survival rate is only $50 \%$.

Treatment options are limited when there is evidence of inoperable metastatic disease. Cytotoxic chemotherapy has a minimal activity and is rarely used (Stadler et al, 2003). Immunotherapy has been demonstrated to provide a modest survival benefit but is associated with high levels of toxicity (Motzer et al, 2002; Coppin et al, 2005). At present, the mainstay of drug therapy for advanced renal cancer involves the sequential use of vascular endothelial growth factor receptor tyrosine kinase inhibitors (such as sunitinib, pazopanib and sorafenib) and inhibitors of mammalian target of rapamycin (such as everolimus or temsirolimus). Despite these recent advances, the outlook for

*Correspondence: T Qayyum, E-mail: tahirl@doctors.org.uk Received I4 May 2012; revised I5 June 2012; accepted 19 June 2012; published online 19 July 2012 these patients remains poor with little prospect of a cure. Sustained efforts continue to identify activated signalling pathways in renal cancer in order to develop further appropriate targeted therapies.

One potential molecular target is the non-receptor tyrosine kinase Src, the first identified human proto-oncogene. Src kinase has a role in signal transduction of multiple oncogenic cellular processes including migration, adhesion, invasion, angiogenesis, proliferation and differentiation and has significant interactions with other cellular proteins such as growth factor receptors (Kopetz et al, 2007). Src kinase is the prototypical member of the Src family kinase (SFK), with a total of eight members expressed in mammalian cells (Src kinase, Blk, Fgr, Fyn Yes, Hck, Lck and Lyn). Src kinase is composed of a C-terminal tail, kinase domain, two protein-protein interaction domains $(\mathrm{SH} 2, \mathrm{SH} 3)$ and a unique amino-terminal domain that varies between Src family members. Src kinase is activated by a number of pathways. Classical activation of Src kinase occurs by an initial dephosphorylation of a conserved tyrosine residue in the $\mathrm{C}$-terminal domain known as the negative regulatory region $\left(\mathrm{Y}^{530}\right)$ and followed by a subsequent autophosphorylation of the $\mathrm{Y}^{419}$ site in the kinase domain (Cooper and Howell, 1993; Engen et al, 2008). Both these events are 
required to occur before the kinase can be considered fully activated. Consequently, antibodies to phosphorylated Src kinase at the $\mathrm{Y}^{419}$ site can be used as a marker for activated Src kinase (Campbell et al, 2008). In addition, when SFK's are activated, several downstream markers such as focal adhesion kinase (FAK) are phosphorylated and could therefore act as biomarkers for SFK activation (Nam et al, 2005). Focal adhesion kinase is phosphorylated at several sites by Src such as $\mathrm{Y}^{397}, \mathrm{Y}^{576}$ and $\mathrm{Y}^{577}$ but it has been reported that the $\mathrm{Y}^{861}$ is the major site of phosphorylation in the carboxyl-terminal domain of FAK (Schaller et al, 1994; Calalb et al, 1995; Calalb et al, 1996).

There is much evidence showing that levels of SFK are elevated in various malignancies such as the prostate, breast, colon and lung (Bolen et al, 1987; Verbeek et al, 1996; Masaki et al, 2003; Tatarov et al, 2009; Elsberger et al, 2010a). Specifically, elevated levels of Src and Lyn (a SFK member) have been observed in breast cancer, and expression of Src was associated with decreased survival (Elsberger et al, 2010a). Src expression does not always correlate with poor survival, it has been reported that Src expression and activity decreases with bladder tumour stage (Fanning et al, 1992; Blaveri et al, 2005; Sanchez-Carbayo et al, 2006; Wu et al, 2009; Thomas et al, 2011) and grade (Qayyum et al, 2012). Expression of Lyn has also been associated with malignancies including the breast, colon and prostate (Bates et al, 2001; Choi et al, 2010).

Previous studies have suggested that the Src kinase family member, Src kinase is involved in the progression of urological tumours (Edwards et al, 2003; Tatarov et al, 2009). Currently Saractinib (AZD05300), an oral Src inhibitor, is being utilised in a phase II trial in renal cancer (COSAK trial).

The aim of the current study is to investigate that whether any of the SFK members are expressed in renal cell carcinoma and to assess whether Src kinase expression and activation status is associated with poor prognosis.

\section{MATERIALS AND METHODS}

A total of 19 clinical specimens were utilised for real-time quantitative PCR (RT-PCR). This consisted of malignant tissue taken from renal cell carcinoma patients at the time of resection. Those specimens were utilised where all tissue was removed at the time of surgery and radiological imaging before surgery showed no evidence of metastatic spread of disease. The Research Ethics Committee of West of Scotland has approved the study.

Real-time quantitative PCR was utilised to determine mRNA expression of the SFK members. At the time of resection, representative parts of malignant tissue were identified, snapfrozen and stored in liquid nitrogen. Total mRNA was extracted from 5-10 mg of renal cell cancer tissue using the TRIZOL method according to manufacturer's protocol (Invitrogen, Paisley UK). RNA quality and quantity was examined by UV spectrometry (GeneQuant analyser, GE Healthcare, Little Chalfont, UK).

Once RNA quality and quantity had been assessed, to ensure that no other DNA was present, DNA-free DNAse treatment and removal reagent kit was added (Applera, Warrington, UK). Before incubation for $30 \mathrm{~min}$ at $37^{\circ} \mathrm{C}$, to ensure that the same volume of cDNA was being utilised for quantification, a starting concentration of $1000 \mathrm{ng}$ of RNA was applied for each sample. Random hexamer primers (50 ng) were used for first-strand cDNA synthesis using SuperScript II RT according to manufacturer's instructions (Invitrogen). Before using cDNA for PCR amplification, 2 units of RNase $\mathrm{H}$ were added to samples and incubated for $20 \mathrm{~min}$ at $37^{\circ} \mathrm{C}$. Real-time quantitative PCR was performed using an ABI Prism 7900 Sequence Detection System (Applied Biosystems, Paisley, UK) and TaqMan Gene Expression Assays. For the TaqMan Gene Expression Assays the manufacturer's protocol with recommended 40 rounds of amplification was applied. Thermal cycler condition
Table I Clinicopathological characteristics of patients utilised for RT-PCR and IHC

\begin{tabular}{|c|c|c|}
\hline & \multicolumn{2}{|c|}{ Numbers } \\
\hline & $\begin{array}{c}\text { RT-PCR } \\
\text { cohort }\end{array}$ & $\begin{array}{l}\text { IHC } \\
\text { cohort }\end{array}$ \\
\hline Age $(<60 />60)$ & $10 / 9$ & $29 / 28$ \\
\hline $\operatorname{Sex}(M / F)$ & $9 / 10$ & $32 / 25$ \\
\hline T stage $(T \mid / T 2 / T 3 / T 4)$ & $5 / 3 / 9 / 2$ & $26 / 12 / 17 / 2$ \\
\hline Grade $(1 / 2 / 3 / 4)$ & $4 / 5 / 6 / 4$ & $10 / 26 / 15 / 6$ \\
\hline Tumour necrosis (absence/presence) & $8 / 11$ & $32 / 25$ \\
\hline Recurrence (no/yes) & $12 / 7$ & $32 / 25$ \\
\hline Tumour volume $\left(<10 \mathrm{~cm}^{3} />10 \mathrm{~cm}^{3}\right)$ & $7 / 12$ & $29 / 28$ \\
\hline
\end{tabular}

were $50{ }^{\circ} \mathrm{C}$ for $2 \mathrm{~min}, 95^{\circ} \mathrm{C}$ for $10 \mathrm{~min}$ followed by $40 \times 95^{\circ} \mathrm{C}$ for $15 \mathrm{~s}$ and $60^{\circ} \mathrm{C}$ for $1 \mathrm{~min}$.

Quantitative values were obtained from the threshold cycle $\left(C_{\mathrm{t}}\right.$ value) at which the increase in TaqMan probe fluorescent signal associated with an exponential increase of each individual PCR product reaching a fixed threshold value. Each individual primer had a fixed threshold $C_{\mathrm{t}}$ value (Table 1). These fixed threshold values were used for every cDNA sample. Negative controls for each primer were included in each run.

To enable the comparison of different mRNA expression levels, their relation to the average expression level of two housekeeping genes (GAPDH, glyceraldehydes-3-phosphate dehydrogenase and HPRT, hypoxanthine-guanine phosphoribosyl transferase) were evaluated. The housekeeping gene with the lowest s.d. (GAPDH) was used for evaluation of the mRNA expression levels. Data were analysed using the Sequence Detection Software (Applied Biosystems), this calculated the threshold cycle $\left(C_{t}\right)$ value. The expression of the target assay was normalised by subtracting the $C_{\mathrm{t}}$ value of the housekeeping gene from the $C_{\mathrm{t}}$ value of the relevant target assay. The fold increase, relative to the control, was obtained by using the formula $2-\Delta C_{\mathrm{t}}$, and then expressed as a percentage $(\times 100)$. All samples were measured in tripiclates.

\section{Statistical analysis}

Differences in expression levels were analysed using the MannWhitney $U$-test or Kruskal-Wallis test, including a Wilcoxon-type test for trends, when appropriate.

Immunohistochemical staining was utilised in a cohort of 57 patients diagnosed with clear cell renal cancer. These patients had undergone complete resection of the tumour at time of nephrectomy and preoperative CT scans showed no evidence of regional or metastatic spread. Patients were staged pathologically and graded according to the TNM classification and Fuhrman grading, respectively. Cancer-specific survival rate was the time from diagnosis until time of death or last follow-up. The cause of death was determined by linkage through the Scottish Cancer Registry. In those who were deceased, if the primary cause of death was of renal cancer, these were classed as cancer-specific and all other causes were non-cancer-specific deaths.

Immunohistochemical staining was utilised to assess expression of c-Src kinase, phosphorylated Src at $\mathrm{Y}^{530}$, phosphorylated Src at $\mathrm{Y}^{419}$ and FAK at the $\mathrm{Y}^{861}$. Both antibodies for dephosphorylated Src and phosphorylated Src are not specific for only c-Src kinase but they also detect other family members including Fyn, Yes and Fgr due to high level of conservation between Src family members.

c-Src kinase and activated Src kinase expression $\left(\mathrm{SrCY}^{419}\right)$ was investigated using antibodies for c-Src kinase (36D10, Cell Signaling Technology, Beverly, MA, USA) and Src $\mathrm{Y}^{416}$ (Cell Signaling Technology). Dephosphorylated Src and FAK were investigated using antibodies for Src $\mathrm{Y}^{527}$ and FAK $\mathrm{Y}^{861}$, 
respectively (Invitrogen). In humans the activated phosphorylation sites that were investigated in the current study are amino acids $\mathrm{Y}^{530}$ and $\mathrm{Y}^{419}$. Antibodies used relate to the rabbit sequence and not the human sequence.

Tissue sections were dewaxed and rehydrated through graded alcohol. Antigen retrieval was performed by heating tissue sections under pressure for $5 \mathrm{~min}$ in a pressure cooker using citrate buffer pH 6 for c-Src kinase, Src $\mathrm{Y}^{527}$, FAK Y ${ }^{861}$ and EDTA buffer $\mathrm{pH} 9$ for Src $\mathrm{Y}^{416}$. Endogenous peroxidase activity was blocked by incubation in $3 \%$ hydrogen peroxide $\left(\mathrm{H}_{2} \mathrm{O}_{2}\right)$. To reduce nonspecific binding the tissue sections were then incubated with $5 \%$ normal horse serum (Vector Laboratories, Burlingame, CA, USA) in antibody dilutent (DAKO Cytomation, Glostrup, Denmark) for $20 \mathrm{~min}$ at room temperature. Incubation with primary antibody was performed with c-Src kinase $(1: 200)$ for $60 \mathrm{~min}$ at room temperature and overnight at $4{ }^{\circ} \mathrm{C}$ for antibodies dephosphorylated Src $\mathrm{Y}^{527}$ (1:3000), phosphorylated Src $\mathrm{Y}^{416}(1: 25)$ and FAK Y $\mathrm{Y}^{861}$ $(1: 200)$. Signal was amplified and visualised using the DAKO Envision Kit (DAKO Cytomation) and the chromagen 3,3'diaminobenzidine (Vector Laboratories). Sections were counterstained, dehydrated and mounted. In each run a positive and negative isotype-matched control was included to ensure no falsepositive staining or intense stromal staining.

\section{Immunohistoscore}

Protein expression for tumour cells was assessed over the full tissue specimen using the weighted histoscore method (Kirkegaard et al, 2006). The weighted histoscore grades staining intensity as negative (0), weak (1), moderate (2) and strong (3), then multiplication of the percentage of tumour cells within each category. Two observers independently scored all tissue sections. Each cellular location was independently assessed for any evidence of expression (Figure 1). Tumours were then divided into those with high (above median) histoscore or low (below or equal to the median) histoscore expression as has previously been reported (Elsberger et al, 2009, 2010b; Qayyum et al, 2012) for the purposes of analysis. Agreement between observers was excellent $(>0.9)$ for all proteins investigated as measured by interclass correlation coefficient.

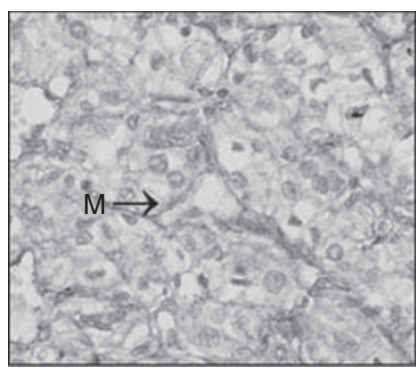

- c-Src
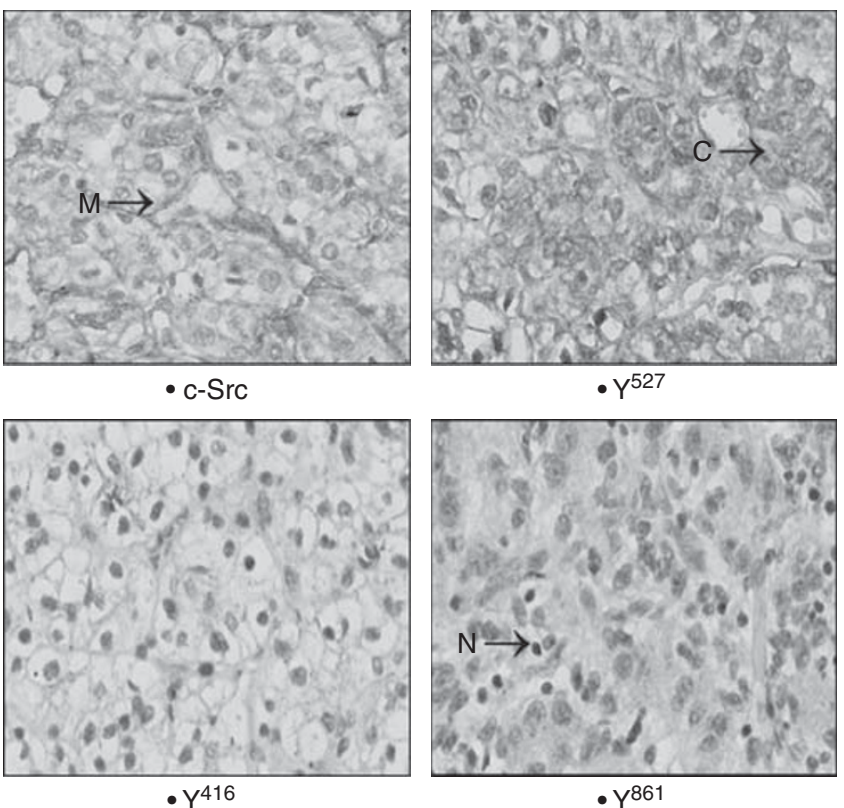

Figure I Representative images of immunohistochemistry for renal cell carcinoma of c-Src kinase, Src $Y^{527}$, Src $Y^{416}$ and FAK $Y^{861}$. Abbreviations: $M=$ membrane staining; $C=$ cytoplasmic staining; $N=$ nuclear staining.
Statistical analysis was undertaken using SPSS (Chicago, IL, USA). Cancer-specific survival rates were generated using the Kaplan-Meier method. The log rank test was utilised to compare significant differences between subset groups using univariate analysis. Multivariate analysis was carried out based on the results of the univariate analysis. Multivariate Cox regression analysis was performed to identify those factors that were independently associated with cancer-specific death. A stepwise backward procedure was utilised to ascertain which of the variables had a significant independent relationship with survival. $\chi^{2}$ analysis was utilised to assess relationships between pathological parameters and the biomarkers at the various locations. Pearson correlation was utilised to assess whether relationships could be identified between the various proteins at the various cellular locations. $P<0.004$ were deemed significant according to Bonferroni's correction.

\section{RESULTS}

The cohort for RT-PCR analysis consisted of 19 renal cell cancers. Median age of diagnosis was 60 years (range 42-72). Table 2 demonstrates clinicopathological parameters of these patients.

The most highly expressed SFK member in renal cell cancer tissue was Src followed by Lyn, Hck, Fgr and Fyn (Table 1, Figure 2). The least expressed SFK member was Blk.

When analysing SFK members in correlation to $\mathrm{T}$ stage, expression of Yes and Blk was significant $(P=0.039, P=0.045$, Figure 3). Interestingly, higher expression of all SFK members was observed in T2-stage disease (Figure 3). Expression of Lck, Lyn, Fyn, Blk and Yes showed a significant decrease between T2 and T3 disease $(P=0.032, P=0.032, P=0.032, P=0.032, P=0.032)$.

When analysing SFK member expression in correlation with tumour grade, no significant difference was observed (Figure 4).

Immunohistochemical analysis was based on 57 clear cell renal cancer patients with full clinical follow-up. Table 2 demonstrates clinicopathological parameters of these patients. Median age at diagnosis was 60 years (range 41-80). Median follow-up was 107 months (range 0.1-163). A total of 22 patients died of their disease. In all, 38 patients had T1/2 disease and 19 patients had T3/4 disease. Tumour volume was recorded for each case; median tumour volume was $9.8 \mathrm{~cm}^{3}$ (range $1-30 \mathrm{~cm}^{3}$ ). Overall, 25 patients subsequently had evidence of recurrence on radiological imaging.

Initial analysis was performed on clinicopathological features, which are known prognostic indicators for survival in renal cancer. $\mathrm{T}$ stage and nuclear grading were significantly associated with poor prognosis, thus demonstrating that this cohort was associated with classical clinical parameters and validating it for use in a biomarker study (Table 3).

Table 2 Intron-skipping primers used for RT-PCR, their fixed threshold $C_{t}$ values and median expression levels in renal cell cancer tissue

\begin{tabular}{|c|c|c|c|}
\hline Gene & $\begin{array}{c}\text { Gene expression } \\
\text { assay ID }\end{array}$ & $\begin{array}{l}\text { Threshold } \\
\left(C_{t}\right) \text { value }\end{array}$ & $\begin{array}{l}\text { Expression } \\
\text { levels }\end{array}$ \\
\hline SRC & Hs00I78494_m I & 0.23 & 404.9 \\
\hline LCK & Hs00 I78427_ml & 0.17 & 13.5 \\
\hline LYN & Hs00176719_ml & 0.25 & 233.3 \\
\hline FYN & Hs00I76628_ml & 0.2 & 53.2 \\
\hline$F G R$ & Hs00I78340_m I & 0.2 & 58.1 \\
\hline HCK & Hs00I76654_ml & 0.26 & 75 \\
\hline$B L K$ & Hs0017644I_ml & 0.19 & 2.5 \\
\hline YES & Hs00736972_ml & 0.26 & 9.7 \\
\hline GAPDH & NA & 0.2 & NA \\
\hline
\end{tabular}

Abbreviations: NA = not applicable; $\mathrm{RT}-\mathrm{PCR}=$ real-time quantitative PCR. 


\section{c-Src kinase}

As c-Src was the highest expressed of all the family members at the mRNA level, expression at the protein level was further investigated. Of the tumours investigated, $98 \%$ showed some degree of nuclear expression, $100 \%$ showed some degree of cytoplasmic expression and $97 \%$ showed some degree of membrane expression. For the purposes of analysis, the median cutoff values of expression at the different cellular locations was utilised, those below and equal to the median score were given a low expression and those above the median were given a score of high as mentioned in the methodology. For nuclear expression this value was 25, cytoplasmic expression was 115 and membrane 100. $\chi^{2}$ analysis demonstrated that cytoplasmic c-Src kinase expression

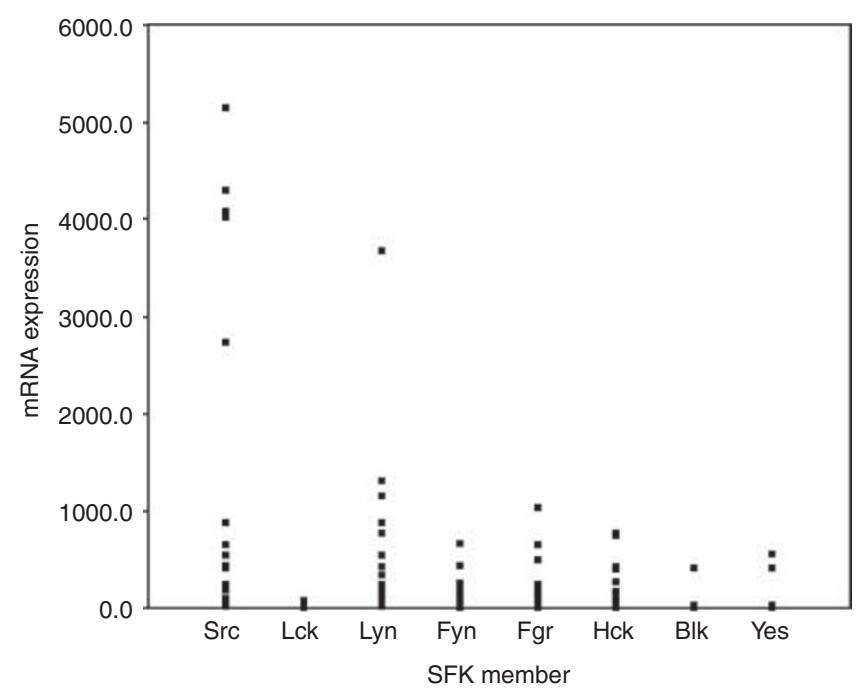

Figure 2 Graph showing expression of mRNA levels of SFK members in renal cancer tissue. positively correlated with nuclear grade $(P=0.023$, Table 4$)$ but no correlation was demonstrated with age, $\mathrm{T}$ stage, tumour necrosis, recurrence or tumour volume. Membrane $\mathrm{c}$-Src kinase protein expression negatively correlated with recurrence $(P=0.021$, Table 4) but no relationship was demonstrated with age, $\mathrm{T}$ stage, nuclear grade, tumour necrosis or tumour volume. These results suggest that membrane c-Src confers good prognosis; however, on univariate analysis expression of $\mathrm{c}-\mathrm{Src}$ at the different cellular locations did not show significance (Table 3). However, a trend was observed between high membrane c-Src kinase expression and improved disease-specific survival, those patients expressing high membrane c-Src kinase had a median survival of 130 months compared with 97 months for those with low expression, this did not however reach significance $(P=0.097$, Figure 5A). On univariate analysis, expression of Src kinase at any of the cellular locations investigated was not significantly associated with disease-specific survival (Table 3). Pearson correlation demonstrated that cytoplasmic c-Src kinase expression demonstrated a positive correlation with nuclear c-Src kinase expression $(P=0.0022$, Table 5).

\section{$\mathrm{Y}^{527}$ Src kinase}

Phosphorylated Src $\mathrm{Y}^{527}$ was investigated. Of the tumours investigated $47 \%$ showed some degree of nuclear expression, $96 \%$ showed some degree of cytoplasmic expression and $93 \%$ showed some degree of membrane expression. There was a difference in the expression of $\mathrm{Y}^{527}$ at the different cellular locations in comparison with c-Src expression with this difference possible as a result of tissue heterogeneity and therefore tissue expression variability as well as c-Src expression encompassing total expression of c-Src in its various phosphorylated forms. The median cutoff values for the purposes of analysis were 0 for nuclear expression, 50 for cytoplasmic and 85 for membrane. $\chi^{2}$ analysis demonstrated that phosphorylated membrane $\mathrm{Y}^{527}$ expression positively correlated with age $(P=0.026$, Table 4$)$, but no correlation was demonstrated with $\mathrm{T}$ stage, nuclear grade, tumour necrosis, recurrence or tumour volume. On univariate
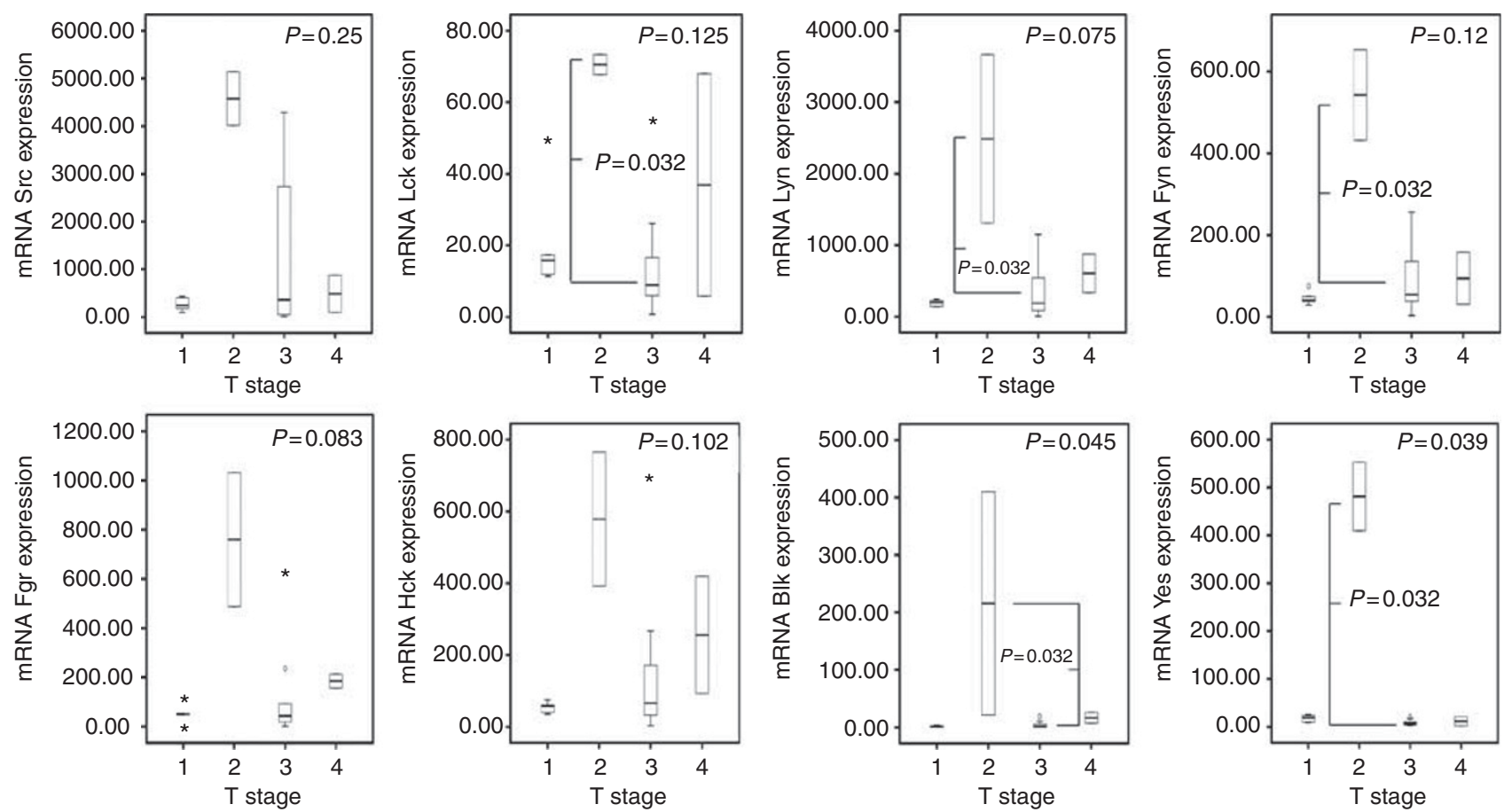

Figure 3 Graphs showing expression of mRNA levels of each SFK member according to T stage. 

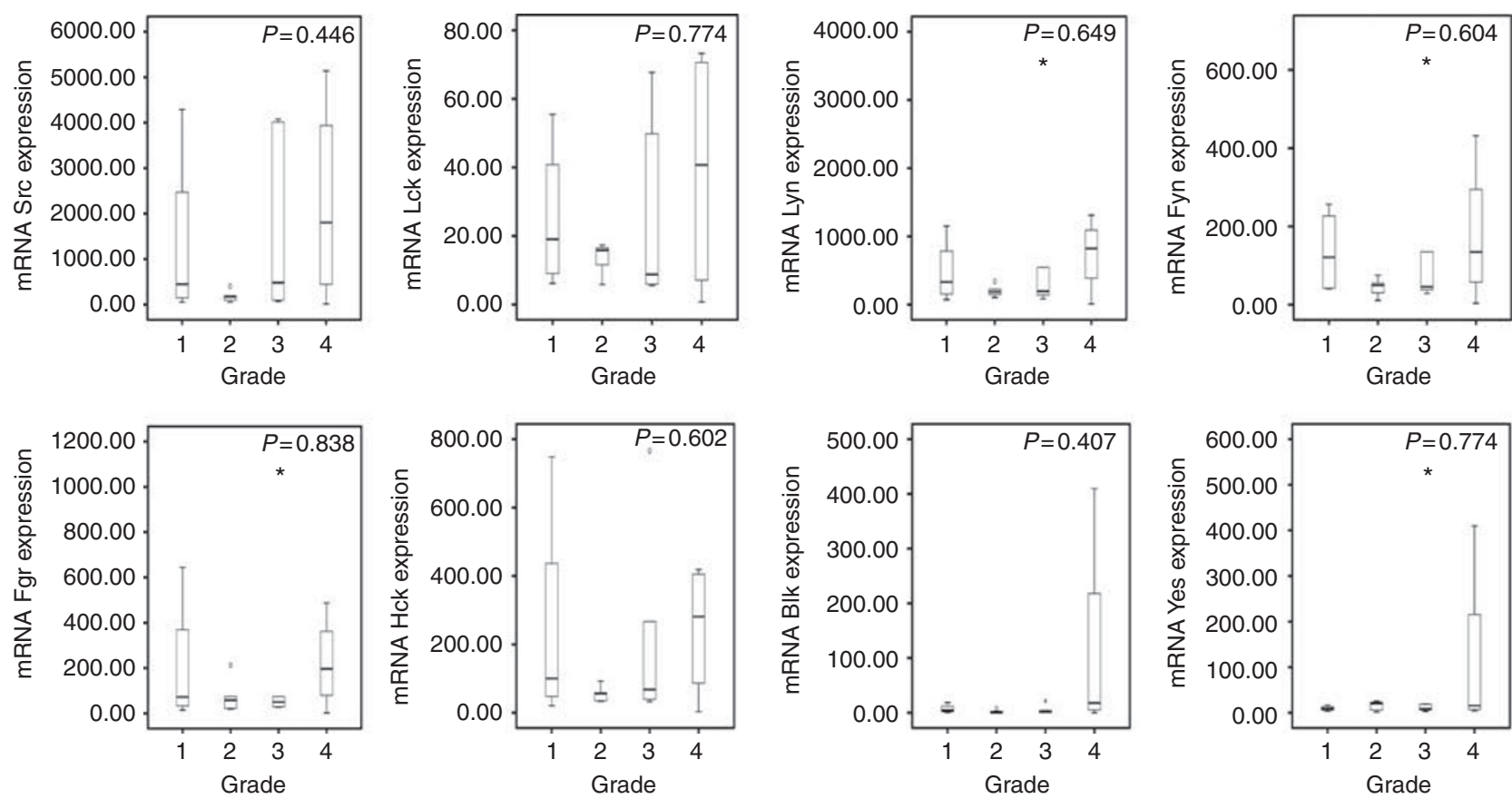

Figure 4 Graphs showing expression of mRNA levels of each SFK member according to tumour grade.

Table 3 Interrelationships between clinicopathological characteristics of patients and protein expression/activation with renal cancer

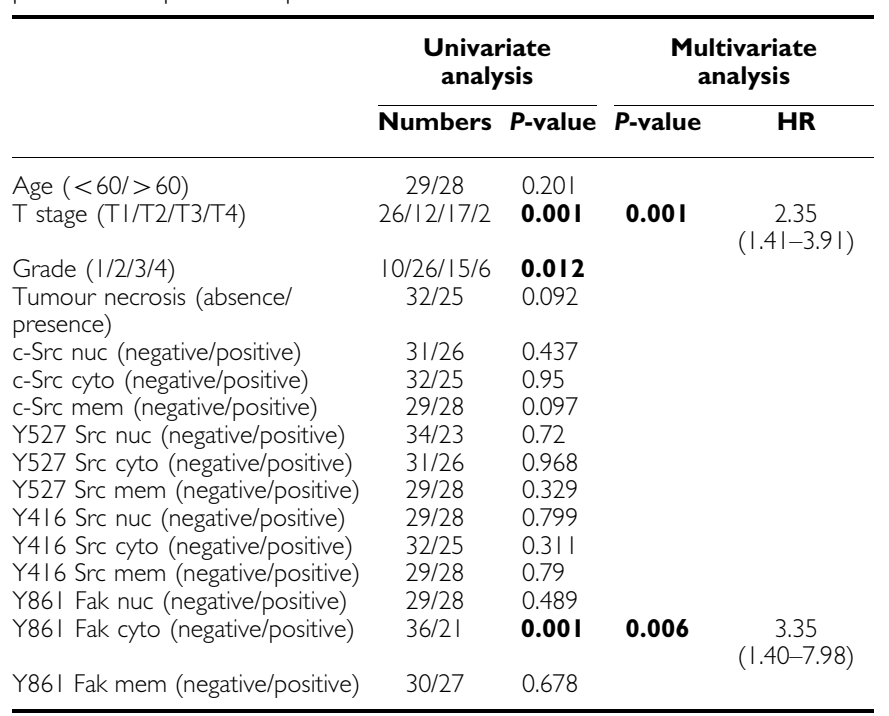

Abbreviation: $\mathrm{HR}=$ hazard ratio. Figures in bold denote significant $P$-values.

analysis, expression of $\mathrm{Y}^{527}$ at any of the cellular locations investigated was not significantly associated with disease-specific survival (Table 3). Pearson correlation demonstrated that phosphorylated cytoplasmic $\mathrm{Y}^{527}$ expression demonstrated a positive correlation with cytoplasmic $c$-Src kinase expression $(P=0.0022$, Table 5).

\section{$\mathrm{Y}^{416}$ Src kinase}

The autophosphorylated status at $\mathrm{Y}^{416}$ was investigated. Of the tumours investigated $84 \%$ showed some degree of nuclear expression, $100 \%$ showed some degree of cytoplasmic expression and $81 \%$ showed some degree of membrane expression. The median cutoff values for the purposes of analysis were 20 for nuclear expression, 100 for cytoplasmic and 20 for membrane. $\chi^{2}$ analysis demonstrated that membrane $\mathrm{Y}^{416}$ expression positively correlated with age $(P=0.026$, Table 4$)$ but no correlation was demonstrated with $\mathrm{T}$ stage, nuclear grade, tumour necrosis, recurrence or tumour volume. On univariate analysis, expression of $\mathrm{Y}^{416}$ at any of the cellular locations investigated was not associated with disease-specific survival (Table 3). Pearson correlation demonstrated that membrane $\mathrm{Y}^{416}$ expression demonstrated a negative correlation with cytoplasmic c-Src kinase expression $(P=0.0005$, Table 5$)$ and a positive correlation was demonstrated with phosphorylated membrane $\mathrm{Y}^{527}$ expression $(P=0.0001$, Table 5).

\section{$\mathrm{Y}^{861}$ FAK}

Of the tumours investigated, $98 \%$ showed some degree of nuclear expression, 39\% showed some degree of cytoplasmic expression and $100 \%$ showed some degree of membrane expression. The median cutoff values for the purposes of analysis were 50 for nuclear expression, 0 for cytoplasmic and 50 for membrane. $\chi^{2}$ analysis demonstrated that expression of cytoplasmic FAK $\mathrm{Y}^{861}$ demonstrated a positive relationship with $\mathrm{T}$ stage, nuclear grade, recurrence and tumour volume $(P=0.023, P=0.001, P=0.036$ and $P=0.045$, respectively, Table 4 ), but no relationship was demonstrated with age or tumour necrosis. On univariate analysis, high expression of cytoplasmic FAK $\mathrm{Y}^{861}$ was associated with decreased cancer-specific survival, $(P=0.001$, Table 3 , Figure 5B). This association was also independent on multivariate analysis (hazard ratio 3.35, 95\% CI 1.40-7.98, $P=0.006$, Table 3). Pearson correlation demonstrated that membrane FAK $\mathrm{Y}^{861}$ expression demonstrated a positive correlation with membrane $\mathrm{Y}^{527}$ and membrane $\mathrm{Y}^{416}$ expression $(P=0.0002$ and $P=0.0002$, Table 5).

\section{DISCUSSION}

We have reported for the first time to our knowledge that SFK members are not only expressed in renal cell carcinoma but are 
The role of Src kinase in renal cancer

$\mathrm{T}$ Qayyum et al

Table 4 Interrelationships between clinicopathological characteristics of patients and protein expression/activation with renal cancer using $\chi^{2}$ analysis

\begin{tabular}{|c|c|c|c|c|c|c|c|}
\hline Variable & Numbers & Age & T stage & Grade & $\begin{array}{l}\text { Tumour necrosis } \\
\text { (absence/presence) }\end{array}$ & $\begin{array}{c}\text { Recurrence } \\
\text { (no/yes) }\end{array}$ & $\begin{array}{c}\text { Tumour volume } \\
\left(<10 \mathrm{~cm}^{3} />10 \mathrm{~cm}^{3}\right)\end{array}$ \\
\hline c-Src nuc (negative/positive) & $31 / 26$ & 0.350 & 0.187 & 0.500 & 0.830 & 0.196 & 0.350 \\
\hline c-Src cyto (negative/positive) & $32 / 25$ & 0.703 & 0.538 & 0.023 & 0.985 & 0.985 & 0.703 \\
\hline c-Src mem (negative/positive) & $29 / 28$ & 0.148 & 0.205 & 0.916 & 0.363 & $0.02 I^{\mathrm{a}}$ & 0.692 \\
\hline Y527 Src nuc (negative/positive) & $34 / 23$ & 0.487 & 0.157 & 0.793 & 0.623 & 0.962 & 0.487 \\
\hline Y527 Src cyto (negative/positive) & $31 / 26$ & 0.144 & 0.447 & 0.708 & 0.751 & 0.749 & 0.684 \\
\hline Y527 Src mem (negative/positive) & $29 / 28$ & 0.026 & 0.335 & 0.916 & 0.882 & 0.358 & 0.513 \\
\hline Y4I6 Src nuc (negative/positive) & $29 / 28$ & 0.692 & 0.889 & 0.916 & 0.227 & 0.701 & 0.513 \\
\hline Y4I 6 Src cyto (negative/positive) & $32 / 25$ & 0.227 & 0.056 & 0.443 & 0.985 & 0.289 & 0.083 \\
\hline Y4I 6 Src mem (negative/positive) & $29 / 28$ & 0.026 & 0.667 & 0.686 & 0.703 & 0.222 & 0.897 \\
\hline Y86। Fak nuc (negative/positive) & $29 / 28$ & 0.897 & 0.478 & 0.482 & 0.703 & 0.701 & 0.692 \\
\hline Y86। Fak cyto (negative/positive) & $36 / 21$ & 0.207 & 0.023 & 0.001 & 0.326 & 0.036 & 0.045 \\
\hline Y86। Fak mem (negative/positive) & $30 / 27$ & 0.150 & 0.702 & 0.753 & 0.540 & 0.933 & 0.361 \\
\hline
\end{tabular}

Figures in bold denote significant $P$-values. ${ }^{a}$ Inverse relationship.
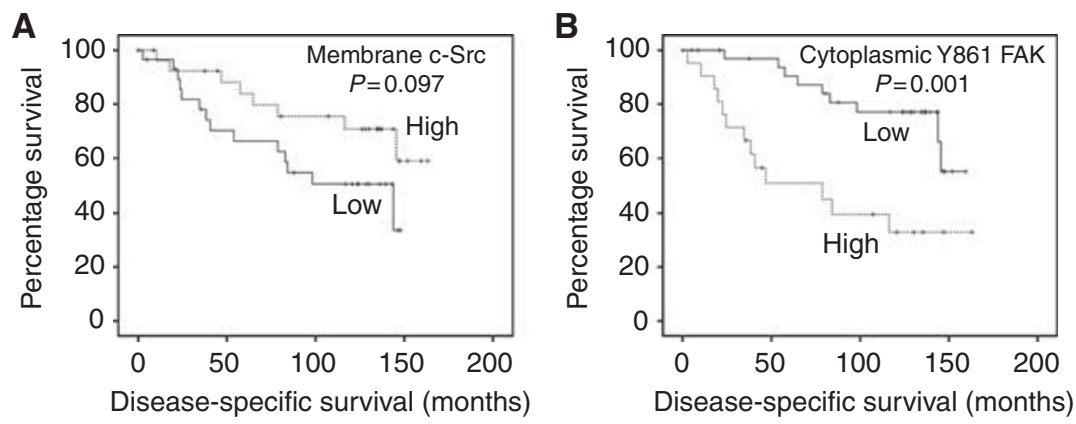

Figure 5. (A) Kaplan-Meier plotted for high and low membrane c-Src kinase expression against disease-specific survival, log rank test $P=0.097$.

(B) Kaplan-Meier plotted for high and low cytoplasmic FAK $Y^{861}$ expression against disease-specific survival, log rank test $P=0.001$.

Table 5 Interrelationships between protein markers at the various cellular locations using Pearson Correlation

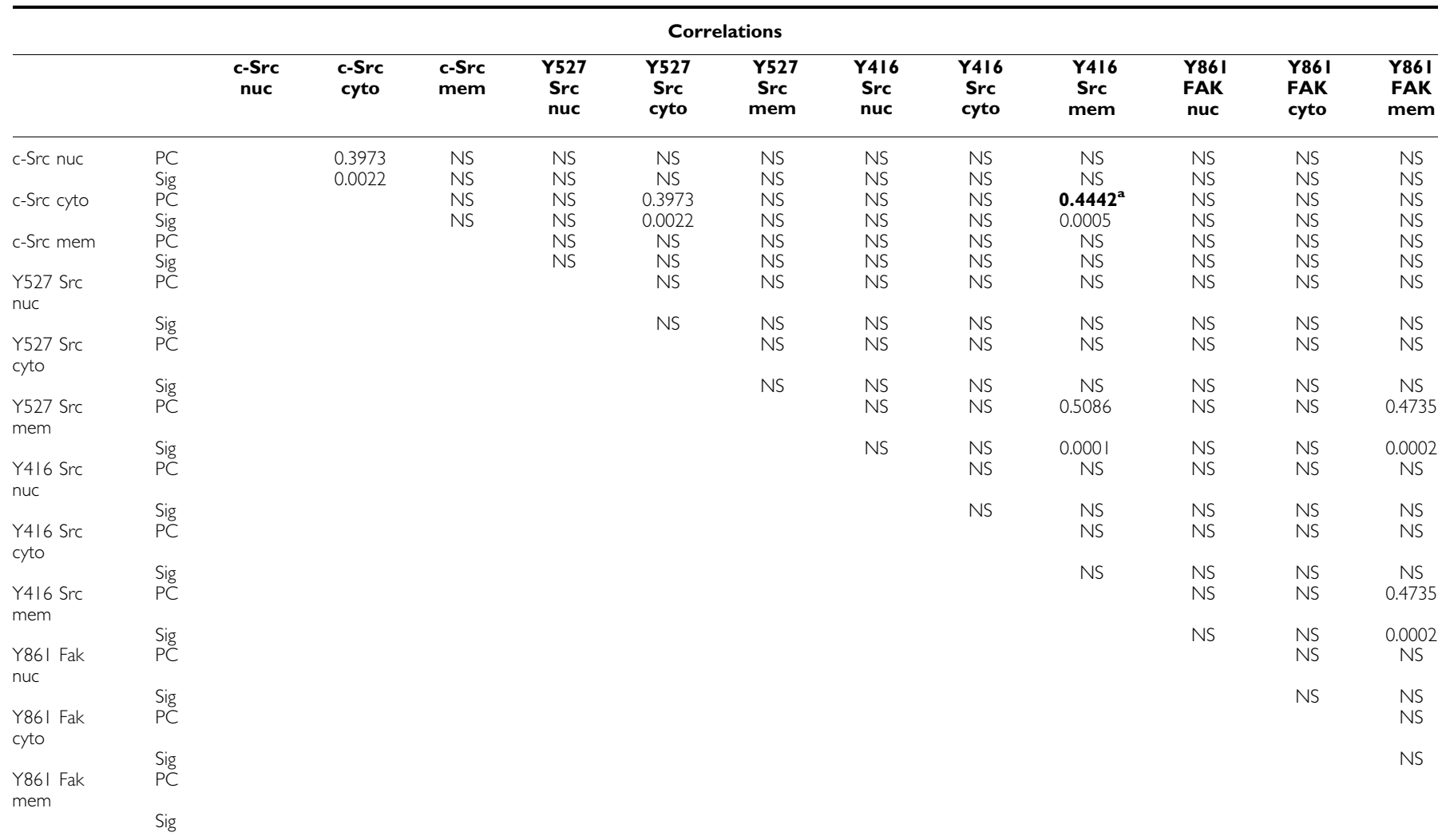

Abbreviations: $\mathrm{PC}=$ Pearson Correlations; Sig = significance (two-tailed); NS = nonsignificant $P$-values. Figure in bold denotes significant $P$-value according to Bonferroni's correction. Inverse relationship. 
expressed to varying degrees. Of the SFK members, Src is the most highly expressed followed by Lyn, Hck, Fgr and Fyn. Src has a five-fold higher expression than the least SFK member expressed. We have also reported that there is a significant fall in expression of Lck, Lyn, Fyn, Blk and Yes between T2 and T3 disease.

Despite having a limited cohort group, we have shown that there is a significant difference in expression of several SFK members between T2 and T3 disease. In renal cancer, T2 disease is still organ confined with T3 disease invading perinephric fascia and/or the adrenal gland. Src kinase has a role in signal transduction of multiple oncogenic cellular processes including migration and invasion. We hypothesise that SFK members are being upregulated at a time when the cancer is going beyond being organ confined and starting to metastasise after which upregulation is no longer required as the cancer has metastasised.

Expressions of SFK members have been demonstrated in a variety of tumours (Bolen et al, 1987; Verbeek et al, 1996; Masaki et al, 2003; Tatarov et al, 2009; Elsberger et al, 2010a). Specifically, expression of Src and Lyn have been demonstrated to be elevated in breast and colon cancer (Bates et al, 2001; Choi et al, 2010; Elsberger et al, 2010a). When assessing activity of SFK members, it has been demonstrated that increased expression of Src is associated with poor survival in breast cancer (Elsberger et al, 2009, 2010a), whereas expression of Src is associated with improved clinical outcome in bladder cancer (Fanning et al, 1992; Thomas et al, 2011; Qayyum et al, 2012).

Given that the cohort size was limited, significant correlations could not be observed between expression of a particular member and survival. Even if a SFK member is expressed, this does not necessarily constitute activity.

Therefore, to gain a better understanding of Src and SFK activation we established a cohort of patient samples to allow us to assess expression and establish correlations with clinical parameters.

To our knowledge, this is the only study investigating the role of Src kinase expression, phosphorylated status $\left(\mathrm{Y}^{527}\right)$, autophosphorylation status $\left(\mathrm{Y}^{419}\right)$ and the downstream marker FAK $\mathrm{Y}^{861}$ in clear cell renal cancer. Src family kinase can be localised at different cellular locations with the inactive form localised at the perinuclear region of cells (Fincham et al, 2000). Much work has been conducted in breast cancer which has reported high cytoplasmic Src kinase levels being associated with shorter disease-specific survival (Wilson et al, 2006) and high expression of activated Src kinase being associated with increased risk of recurrence (Elsberger et al, 2009). In addition, prostate cancer studies have demonstrated that Src kinase expression and activation is associated in quicker time to relapse and shorter disease-specific survival (Tatarov et al, 2009). Therefore, current literature supports a role for $\mathrm{Src}$ as a negative prognostic marker in solid tumours.

In contrast to the current study, $\chi^{2}$ analysis demonstrates that membrane Src kinase expression was negatively associated with recurrence and a trend towards improved survival was also observed. These results require confirming in a larger patient cohort; however, the combination of reduced recurrence and improved survival being associated with Src kinase expression suggests that Src kinase might not be responsible for driving progression in renal cancer. Although Src expression itself might not be involved with renal cancer progression, activation of the Src or other Src family members might be involved.

\section{REFERENCES}

Bates RC, Edwards NS, Burns GF, Fisher DE (2001) A CD44 survival pathway triggers chemoresistance via lyn kinase and phosphoinositide 3-kinase/Akt in colon carcinoma cells. Cancer Res 61(13): 5275-5283
It has been suggested that a biomarker for prediction of Src kinase activity would be to measure phosphorylation of the protein at a site associated with activity (Bolen et al, 1987; Masaki et al, 1998). Currently, there are two sites within Src known to be associated with activation. Phosphorylation of the tyrosine residue 530 on the c-terminal tail by Csk tyrosine kinase acts as a negative regulatory protein-binding site, keeping Src kinase in a closed confirmation (Roskoski Jr, 2005). Upon dephosphorylation this allows Src kinase to undergo a transformational change allowing the second site of activation, $\mathrm{Y}^{419}$, to be accessible, which needs to be autophosphorylated before full activation of Src kinase. $\mathrm{Y}^{419}$ is referred to as the classical site and is the most commonly used in cell line studies investigating the functional relevance of Src kinase activation (Finn et al, 2007). In this study, membrane $\mathrm{Y}^{416}$ expression demonstrated a negative correlation with cytoplasmic Src kinase and a positive correlation with cytoplasmic $\mathrm{Y}^{527}$, confirming validity of results regarding Src kinase needing to undergo dephosphorylation at the $\mathrm{Y}^{530}$ before autophosphorylation at the $\mathrm{Y}^{419}$ can proceed. Activated phosphorylated Src kinase at the classical site $\mathrm{Y}^{416}$ when assessed at individual locations was shown to have no correlation to disease-specific survival. This antibody is not specific for one particularly Src family member as this part of the sequence is highly conserved between Src family members making it impossible to produce an antibody specific for only one member. Therefore, as the antibody cross reacts with all family members that are phosphorylated at this site, this could account for the lack of correlation with disease-specific survival.

Autophosphorylation of FAK at the $\mathrm{Y}^{397}$ site occurs as a result of many stimuli, thereby creating a high affinity binding site for the Src homology 2 domain of several proteins including Src kinase (Schaller et al, 1994; Calalb et al, 1995). Src phosphorylates FAK at several sites but it has been reported that the $\mathrm{Y}^{861}$ is the major site on the FAK domain associated with activation (Calalb et al, 1996), and therefore may be used as a surrogate marker not only for SFK activation but also for SFK function (Nam et al, 2005).

Expression of cytoplasmic $\mathrm{Y}^{861}$ was associated with reduced disease-specific survival and positively correlated with $\mathrm{T}$ stage, nuclear grade, recurrence and tumour volume, suggesting that the phosphorylation of $\mathrm{Y}^{861}$ may be associated with more aggressive renal cancers (Table 3). Expression of cytoplasmic $\mathrm{Y}^{861}$ positively correlated with membrane $\mathrm{Y}^{416}$, further suggesting that of the Src kinase family members, c-Src kinase may not be responsible for the phosphorylation at the $\mathrm{Y}^{861}$ site in clear cell renal cancer, therefore implicating a role for an alternative SFK member in renal cancer progression.

The current study has demonstrated that c-Src is the most highly expressed SFK member in renal cell cancer. Activation of c-Src kinase itself is associated with improved survival but the presence of the downstream marker FAK, itself a surrogate marker for SFK member activation, is associated with decreased cancerspecific survival, suggesting that another SFK member is responsible for poor survival in renal cell cancer.

\section{ACKNOWLEDGEMENTS}

This work was supported by Think Pink, Gartnavel Urology Endowment Fund and Renal Cancer Research Fund.
Blaveri E, Simko JP, Korkola JE, Brewer JL, Baehner F, Mehta K, Devries S, Koppie T, Pejavar S, Carroll P, Waldman FM (2005) Bladder cancer outcome and subtype classification by gene expression. Clin Cancer Res 11(11): 4044-4055 
Bolen JB, Veillette A, Schwartz AM, DeSeau V, Rosen N (1987) Activation of pp60c-src protein kinase activity in human colon carcinoma. Proc Natl Acad Sci USA 84(8): 2251-2255

Calalb MB, Polte TR, Hanks SK (1995) Tyrosine phosphorylation of focal adhesion kinase at sites in the catalytic domain regulates kinase activity: a role for Src family kinases. Mol Cell Biol 15(2): 954-963

Calalb MB, Zhang X, Polte TR, Hanks SK (1996) Focal adhesion kinase tyrosine-861 is a major site of phosphorylation by Src. Biochem Biophys Res Commun 228(3): 662-668

Campbell EJ, McDuff E, Tatarov O, Tovey S, Brunton V, Cooke TG, Edwards J (2008) Phosphorylated c-Src in the nucleus is associated with improved patient outcome in ER-positive breast cancer. $\mathrm{Br} \mathrm{J}$ Cancer 99(11): 1769-1774

Choi YL, Bocanegra M, Kwon MJ, Shin YK, Nam SJ, Yang JH, Kao J, Godwin AK, Pollack JR (2010) LYN is a mediator of epithelialmesenchymal transition and a target of dasatinib in breast cancer. Cancer Res 70(6): 2296-2306

Cooper JA, Howell B (1993) The when and how of Src regulation. Cell 73(6): 1051-1054

Coppin C, Porzsolt F, Awa A, Kumpf J, Coldman A, Wilt T (2005) Immunotherapy for advanced renal cell cancer. Cochrane Database Syst Rev (1): CD001425

Edwards J, Krishna NS, Witton CJ, Bartlett JM (2003) Gene amplifications associated with the development of hormone-resistant prostate cancer. Clin Cancer Res 9(14): 5271-5281

Elsberger B, Fullerton R, Zino S, Jordan F, Mitchell TJ, Brunton VG, Mallon EA, Shiels PG, Edwards J (2010a) Breast cancer patients' clinical outcome measures are associated with Src kinase family member expression. $\mathrm{Br} J$ Cancer 103(6): 899-909

Elsberger B, Tan BA, Mallon EA, Brunton VG, Edwards J (2010b) Is there an association with phosphorylation and dephosphorylation of Src kinase at tyrosine 530 and breast cancer patient disease-specific survival. Br J Cancer 103(12): 1831-1834

Elsberger B, Tan BA, Mitchell TJ, Brown SB, Mallon EA, Tovey SM, Cooke TG, Brunton VG, Edwards J (2009) Is expression or activation of Src kinase associated with cancer-specific survival in ER-, PR- and HER2-negative breast cancer patients? Am J Pathol 175(4): 1389-1397

Engen JR, Wales TE, Hochrein JM, Meyn III MA, Banu OS, Bahar I, Smithgall TE (2008) Structure and dynamic regulation of Src-family kinases. Cell Mol Life Sci 65(19): 3058-3073

Fanning P, Bulovas K, Saini KS, Libertino JA, Joyce AD, Summerhayes IC (1992) Elevated expression of pp60c-src in low grade human bladder carcinoma. Cancer Res 52(6): 1457-1462

Fincham VJ, Brunton VG, Frame MC (2000) The SH3 domain directs acto-myosin-dependent targeting of $\mathrm{v}$-Src to focal adhesions via phosphatidylinositol 3-kinase. Mol Cell Biol 20(17): 6518-6536

Finn RS, Dering J, Ginther C, Wilson CA, Glaspy P, Tchekmedyian N, Slamon DJ (2007) Dasatinib, an orally active small molecule inhibitor of both the src and abl kinases, selectively inhibits growth of basal-type/ "triple-negative" breast cancer cell lines growing in vitro. Breast Cancer Res Treat 105(3): 319-326

Kirkegaard T, Edwards J, Tovey S, McGlynn LM, Krishna SN, Mukherjee R, Tam L, Munro AF, Dunne B, Bartlett JM (2006) Observer variation in immunohistochemical analysis of protein expression, time for a change? Histopathology 48(7): 787-794
Kopetz S, Shah AN, Gallick GE (2007) Src continues aging: current and future clinical directions. Clin Cancer Res 13(24): 7232-7236

Masaki T, Igarashi K, Tokuda M, Yukimasa S, Han F, Jin YJ, Li JQ, Yoneyama H, Uchida N, Fujita J, Yoshiji H, Watanabe S, Kurokohchi K, Kuriyama S (2003) pp60c-src activation in lung adenocarcinoma. Eur J Cancer 39(10): 1447-1455

Masaki T, Okada M, Shiratori Y, Rengifo W, Matsumoto K, Maeda S, Kato N, Kanai F, Komatsu Y, Nishioka M, Omata M (1998) pp60c-src activation in hepatocellular carcinoma of humans and LEC rats. Hepatology 27(5): 1257-1264

Motzer RJ, Bacik J, Murphy BA, Russo P, Mazumdar M (2002) Interferon-alfa as a comparative treatment for clinical trials of new therapies against advanced renal cell carcinoma. J Clin Oncol 20(1): 289-296

Nam S, Kim D, Cheng JQ, Zhang S, Lee JH, Buettner R, Mirosevich J, Lee FY, Jove R (2005) Action of the Src family kinase inhibitor, dasatinib (BMS-354825), on human prostate cancer cells. Cancer Res 65(20): 9185-9189

Qayyum T, Fyffe G, Duncan M, McArdle PA, Hilmy M, Orange C, Halbert G, Seywright M, Horgan PG, Underwood MA, Edwards J (2012) The interrelationships between Src, Cav-1 and RhoGD12 in transitional cell carcinoma of the bladder. Br J Cancer 106(6): 1187-1195

Roskoski Jr. R (2005) Src kinase regulation by phosphorylation and dephosphorylation. Biochem Biophys Res Commun 331(1): 1-14

Sanchez-Carbayo M, Socci ND, Lozano J, Saint F, Cordon-Cardo C (2006) Defining molecular profiles of poor outcome in patients with invasive bladder cancer using oligonucleotide microarrays. J Clin Oncol 24(5): 778-789

Schaller MD, Hildebrand JD, Shannon JD, Fox JW, Vines RR, Parsons JT (1994) Autophosphorylation of the focal adhesion kinase, pp125FAK, directs SH2-dependent binding of pp60src. Mol Cell Biol 14(3): 1680-1688

Stadler WM, Huo D, George C, Yang X, Ryan CW, Karrison T, Zimmerman TM, Vogelzang NJ (2003) Prognostic factors for survival with gemcitabine plus 5-fluorouracil based regimens for metastatic renal cancer. J Urol 170(4 Part 1): 1141-1145

Tatarov O, Mitchell TJ, Seywright M, Leung HY, Brunton VG, Edwards J (2009) SRC family kinase activity is up-regulated in hormone-refractory prostate cancer. Clin Cancer Res 15(10): 3540-3549

Thomas S, Overdevest JB, Nitz MD, Williams PD, Owens CR, SanchezCarbayo M, Frierson HF, Schwartz MA, Theodorescu D (2011) Src and caveolin-1 reciprocally regulate metastasis via a common downstream signaling pathway in bladder cancer. Cancer Res 71(3): 832-841

Verbeek BS, Vroom TM, Adriaansen-Slot SS, Ottenhoff-Kalff AE, Geertzema JG, Hennipman A, Rijksen G (1996) c-Src protein expressi on is increased in human breast cancer. An immunohistochemical and biochemical analysis. J Pathol 180(4): 383-388

Wilson GR, Cramer A, Welman A, Knox F, Swindell R, Kawakatsu H, Clarke RB, Dive C, Bundred NJ (2006) Activated c-SRC in ductal carcinoma in situ correlates with high tumour grade, high proliferation and HER2 positivity. Br I Cancer 95(10): 1410-1414

Wu Y, Moissoglu K, Wang H, Wang X, Frierson HF, Schwartz MA, Theodorescu D (2009) Src phosphorylation of RhoGDI2 regulates its metastasis suppressor function. Proc Natl Acad Sci USA 106(14): 5807-5812

This work is published under the standard license to publish agreement. After 12 months the work will become freely available and the license terms will switch to a Creative Commons Attribution-NonCommercial-Share Alike 3.0 Unported License. 